CUBO, A Mathematical Journal

Vol.22, $\mathrm{N}^{\circ} 02,(233-255)$. August 2020

Received: 23 May, 2020 | Accepted: 20 July, 2020

\title{
Hyers-Ulam stability of an additive-quadratic functional equation
}

\author{
Vediyappan Govindan ${ }^{1}$, ChOONKIL PARK ${ }^{2}$, \\ SANDra Pinelas ${ }^{3}$ and Themistocles M. Rassias ${ }^{4}$ \\ 1 Department of Mathematics, Sri Vidya Mandir Arts $\&$ Science College, Katteri, India. \\ ${ }^{2}$ Research Institute of Natural Sciences, Hanyang University, Seoul-04763, Korea. \\ 3 Departamento de Ciências Exatas e Engenharia, Academia Militar, Portugal. \\ 4 Department of Mathematics, National Technical University of Athens, Greece. \\ govindoviya@gmail.com, baak@hanyang.ac.kr, \\ sandra.pinelas@gmail.com, trassias@math.ntua.gr
}

\section{ABSTRACT}

In this paper, we introduce the following $(a, b, c)$-mixed type functional equation of the form

$g\left(a x_{1}+b x_{2}+c x_{3}\right)-g\left(-a x_{1}+b x_{2}+c x_{3}\right)+g\left(a x_{1}-b x_{2}+c x_{3}\right)-g\left(a x_{1}+b x_{2}-c x_{3}\right)+$ $2 a^{2}\left[g\left(x_{1}\right)+g\left(-x_{1}\right)\right]+2 b^{2}\left[g\left(x_{2}\right)+g\left(-x_{2}\right)\right]+2 c^{2}\left[g\left(x_{3}\right)+g\left(-x_{3}\right)\right]+a\left[g\left(x_{1}\right)-g\left(-x_{1}\right)\right]+$ $b\left[g\left(x_{2}\right)-g\left(-x_{2}\right)\right]+c\left[g\left(x_{3}\right)-g\left(-x_{3}\right)\right]=4 g\left(a x_{1}+c x_{3}\right)+2 g\left(-b x_{2}\right)+2 g\left(b x_{2}\right)$

where $a, b, c$ are positive integers with $a>1$, and investigate the solution and the Hyers-Ulam stability of the above functional equation in Banach spaces by using two different methods.

\section{RESUMEN}

En este artículo introducimos la siguiente ecuación funcional de tipo $(a, b, c)$-mixta de la forma

$g\left(a x_{1}+b x_{2}+c x_{3}\right)-g\left(-a x_{1}+b x_{2}+c x_{3}\right)+g\left(a x_{1}-b x_{2}+c x_{3}\right)-g\left(a x_{1}+b x_{2}-c x_{3}\right)+$ $2 a^{2}\left[g\left(x_{1}\right)+g\left(-x_{1}\right)\right]+2 b^{2}\left[g\left(x_{2}\right)+g\left(-x_{2}\right)\right]+2 c^{2}\left[g\left(x_{3}\right)+g\left(-x_{3}\right)\right]+a\left[g\left(x_{1}\right)-g\left(-x_{1}\right)\right]+$ $b\left[g\left(x_{2}\right)-g\left(-x_{2}\right)\right]+c\left[g\left(x_{3}\right)-g\left(-x_{3}\right)\right]=4 g\left(a x_{1}+c x_{3}\right)+2 g\left(-b x_{2}\right)+2 g\left(b x_{2}\right)$

donde $a, b, c$ son enteros positivos con $a>1$, e investigamos la solución y la estabilidad de Hyers-Ulam de la ecuación funcional anterior en espacios de Banach usando dos métodos diferentes.

Keywords and Phrases: Hyers-Ulam stability, mixed type functional equation, Banach space, fixed point.

2020 AMS Mathematics Subject Classification: 39B52, 32B72, 32B82. 


\section{Introduction}

The stability problem of functional equations originated form a question of Ulam [28] concerning the stability of group homomorphisms. Hyers [12] gave a first affirmative partial answer to the question of Ulam [28 for Banach spaces. Hyers theorem was generalized by Aoki [3] for additive mappings and Rassias [12] for quadratic mappings. During the last three decades the stability theorem of Rassias [26] provided a lot of influence for the development of stability theory of a large variety of functional equations (see [1, 2, 4, 1, 9, 11, 14, 17, 18, 21, 22, 23, 27]). One of the most famous functional equations is the following additive functional equation

$$
g(x+y)=g(x)+g(y)
$$

In 1821, it was first solved by Cauchy in the class of continuous real-valued functions. It is often called Cauchy additive functional equation in honour of Cauchy. The theory of additive functional equations is frequently applied to the development of theories of other functional equations. Moreover, the properties of additive functional equations are powerful tools in almost every field of natural and social science ([6, 24, 26]). Every solution of the additive functional equation (1.1) is called an additive mapping.

The function $g(x)=x^{2}$ satisfies the functional equation

$$
g(x+y)+g(x-y)=2 g(x)+2 g(y)
$$

and therefore, the functional equation (1.2) is called quadratic functional equation. The HyersUlam stability theorem for the quadratic functional equation (1.2) was proved by Skof [25] for the mapping $g: E_{1} \rightarrow E_{2}$, where $E_{1}$ is a normed space and $E_{2}$ is a Banach space.

Moslehian and Rassias [20] studied the Hyers-Ulam stability problem in non-Archimedean normed spaces. Mirzavaziri and Moslehian [19] studied the Hyers-Ulam stability of a quadratic functional equation in Banach spaces by using the fixed point method and Ciepliński [5] surveyed the Hyers-Ulam stability of functional equations by using the fixed point method. Ebadian, Ghobadipour and Eshaghi Gordji [8] proved the Hyers-Ulam stability of bimultipliers and Jordan bimultipliers in $C^{*}$-ternary algebras by using the fixed point method for a three variable additive functional equation.

Motivated by Ebadian et al. [8], we introduce the following three variable generalized additivequadratic functional equation of the form $D g\left(x_{1}, x_{2}, x_{3}\right):=$

$$
\begin{gathered}
g\left(a x_{1}+b x_{2}+c x_{3}\right)-g\left(-a x_{1}+b x_{2}+c x_{3}\right)+g\left(a x_{1}-b x_{2}+c x_{3}\right)-g\left(a x_{1}+b x_{2}-c x_{3}\right) \\
+2 a^{2}\left[g\left(x_{1}\right)+g\left(-x_{1}\right)\right]+2 b^{2}\left[g\left(x_{2}\right)+g\left(-x_{2}\right)\right]+2 c^{2}\left[g\left(x_{3}\right)+g\left(-x_{3}\right)\right] \\
+a\left[g\left(x_{1}\right)-g\left(-x_{1}\right)\right]+b\left[g\left(x_{2}\right)-g\left(-x_{2}\right)\right]+c\left[g\left(x_{3}\right)-g\left(-x_{3}\right)\right]
\end{gathered}
$$




$$
-\left[4 g\left(a x_{1}+c x_{3}\right)+2 g\left(-b x_{2}\right)+2 g\left(b x_{2}\right)\right]=0
$$

where $a, b, c$ are positive integers with $a>1$, and investigate the solution and the Hyers-Ulam stability of the three variable generalized additive-quadratic functional equation (1.3) in Banach spaces by using the direct method and the fixed point method.

\section{Solution of the functional equation (1.3): when $g$ is odd}

In this section, we investigate the solution of the functional equation (1.3) for an odd mapping case. Throughout this section, let $X$ and $Y$ be real vector spaces.

Theorem 1. If an odd mapping $g: X \rightarrow Y$ satisfies the functional equation (1.1) if and only if $g: X \rightarrow Y$ satisfies the functional equation (1.3).

Proof. Assume that $g: X \rightarrow Y$ satisfies the functional equation (1.1).

Since $g$ is odd, $g(0)=0$.

Replacing $(x, y)$ by $(x, x)$ and by $(x, 2 x)$ respectively in (1.1), we obtain

$$
g(2 x)=2 g(x) \text { and } g(3 x)=3 g(x)
$$

for all $x \in X$. In general for any positive integer $d$, we have

$$
g(d x)=d g(x)
$$

for all $x \in X$. It is easy to verify from (1.1) that

$$
g\left(d^{2} x\right)=d^{2} g(x) \text { and } g\left(d^{3} x\right)=d^{3} g(x)
$$

for all $x \in X$. Replacing $(x, y)$ by $\left(a x_{1}+b x_{2}, c x_{3}\right)$ in (1.1), we get

$$
g\left(a x_{1}+b x_{2}+c x_{3}\right)=g\left(a x_{1}+b x_{2}\right)+g\left(c x_{3}\right)
$$

for $x_{1}, x_{2}, x_{3} \in X$. Replacing $x_{1}$ by $-x_{1}$ in (2.4), we get

$$
g\left(-a x_{1}+b x_{2}+c x_{3}\right)=g\left(-a x_{1}+b x_{2}\right)+g\left(c x_{3}\right)
$$

for $x_{1}, x_{2}, x_{3} \in X$. Replacing $x_{2}$ by $-x_{2}$ in (2.4), we have

$$
g\left(a x_{1}-b x_{2}+c x_{3}\right)=g\left(a x_{1}-b x_{2}\right)+g\left(c x_{3}\right)
$$

for $x_{1}, x_{2}, x_{3} \in X$. Replacing $x_{3}$ by $-x_{3}$ in (2.4), we obtain

$$
g\left(a x_{1}+b x_{2}-c x_{3}\right)=g\left(a x_{1}+b x_{2}\right)+g\left(-c x_{3}\right)
$$


for $x_{1}, x_{2}, x_{3} \in X$. By (2.4), (2.5), (2.6), (2.7), (1.1) and (2.3), we get

$$
\begin{array}{r}
g\left(a x_{1}+b x_{2}+c x_{3}\right)-g\left(-a x_{1}+b x_{2}+c x_{3}\right)+g\left(a x_{1}-b x_{2}+c x_{3}\right)-g\left(a x_{1}+b x_{2}-c x_{3}\right) \\
=2 a g\left(x_{1}\right)-2 b g\left(x_{2}\right)+2 c g\left(x_{3}\right)
\end{array}
$$

for $x_{1}, x_{2}, x_{3} \in X$. Adding $2 a g\left(x_{1}\right)-2 b g\left(x_{2}\right)+2 c g\left(x_{3}\right)+2 a^{2} g\left(x_{1}\right)+2 b^{2} g\left(x_{2}\right)+2 c^{2} g\left(x_{3}\right)$ to both sides and using the oddness of $g$, we get (1.3).

Conversely, assume that $g$ satisfies (1.3). Letting $x_{3}=0$ in (1.3), we have

$$
\begin{aligned}
& g\left(a x_{1}+b x_{2}+c x_{3}\right)-g\left(-a x_{1}+b x_{2}+c x_{3}\right)+g\left(a x_{1}-b x_{2}+c x_{3}\right)-g\left(a x_{1}+b x_{2}-c x_{3}\right) \\
&+2 a^{2}\left[g\left(x_{1}\right)+g\left(-x_{1}\right)\right]+ 2 b^{2}\left[g\left(x_{2}\right)+g\left(-x_{2}\right)\right]+2 c^{2}\left[g\left(x_{3}\right)+g\left(-x_{3}\right)\right]+a\left[g\left(x_{1}\right)-g\left(-x_{1}\right)\right] \\
&+b\left[g\left(x_{2}\right)-g\left(-x_{2}\right)\right]+c\left[g\left(x_{3}\right)-g\left(-x_{3}\right)\right] \\
&=2 g\left(a x_{1}-b x_{2}\right)+2 a g\left(x_{1}\right)+2 b g\left(x_{2}\right)
\end{aligned}
$$

for all $x_{1}, x_{2} \in X$, since $g$ is odd. So

$$
2 g\left(a x_{1}-b x_{2}\right)+2 a g\left(x_{1}\right)+2 b g\left(x_{2}\right)=4 g\left(a x_{1}\right)
$$

for all $x_{1}, x_{2} \in X$. Letting $x_{2}=0$ in (2.9), we have $2 g\left(a x_{1}\right)+2 a g\left(x_{1}\right)=4 g\left(a x_{1}\right)$ and so $g\left(a x_{1}\right)=$ $a g\left(x_{1}\right)$ for all $x_{1} \in X$. Letting $x_{1}=0$ in (2.9), we have $-2 g\left(b x_{2}\right)+2 b g\left(x_{2}\right)=0$ and so $g\left(b x_{2}\right)=$ $b g\left(x_{2}\right)$ for all $x_{2} \in X$. It follows from (2.9) that

$$
2 g\left(a x_{1}-b x_{2}\right)+2 g\left(a x_{1}\right)+2 g\left(b x_{2}\right)=4 g\left(a x_{1}\right)
$$

for all $x_{1}, x_{2} \in X$ and so

$$
g(x-y)+g(y)=g(x)
$$

for all $x, y \in X$. Letting $z=x-y$ in the above equation, we get $g(z)+g(y)=g(z+y)$ for all $z, y \in X$.

\section{Solution of the functional equation (1.3): when $g$ is even}

In this section, we investigate the solution of the functional equation (1.3) for an even mapping case. Throughout this section, let $X$ and $Y$ to be real vector spaces.

Theorem 2. If an even mapping $g: X \rightarrow Y$ satisfies the functional equation (1.2) if and only if $g: X \rightarrow Y$ satisfies the functional equation (1.3).

Proof. Assume that $g: X \rightarrow Y$ satisfies the functional equation (1.2).

Setting $x=y=0$ in (1.2), we get $g(0)=0$. 
Replacing $(x, y)$ by $(x, x)$ and by $(x, 2 x)$, respectively, in (1.2), we obtain

$$
g(2 x)=4 g(x) \text { and } g(3 x)=9 g(x)
$$

for all $x \in X$. In general for any positive integer $d$, we have

$$
g(d x)=d^{2} g(x)
$$

for all $x \in X$. It is easy to verify from (1.2) that

$$
g\left(d^{2} x\right)=d^{4} g(x) \text { and } g\left(d^{3} x\right)=d^{6} g(x)
$$

for all $x \in X$. Replacing $(x, y)$ by $\left(a x_{1}, c x_{3}\right)$ in (1.2), we get

$$
g\left(a x_{1}+c x_{3}\right)+g\left(a x_{1}-c x_{3}\right)=2 g\left(a x_{1}\right)+2 g\left(c x_{3}\right)
$$

for $x_{1}, x_{2}, x_{3} \in X$.

Multiplying 2 on both sides and using (3.3), we get

$$
2 g\left(a x_{1}+c x_{3}\right)+2 g\left(a x_{1}-c x_{3}\right)=4 a^{2} g\left(x_{1}\right)+4 c^{2} g\left(x_{3}\right)
$$

for $x_{1}, x_{2}, x_{3} \in X$.

Adding $2 g\left(a x_{1}+c x_{3}\right)$ to (3.5) on both sides and using (3.3), we obtain

$$
2 g\left(a x_{1}+c x_{3}\right)+2 g\left(a x_{1}-c x_{3}\right)+2 g\left(a x_{1}+c x_{3}\right)=4 a^{2} g\left(x_{1}\right)+4 c^{2} g\left(x_{3}\right)+2 g\left(a x_{1}+c x_{3}\right)
$$

for $x_{1}, x_{2}, x_{3} \in X$. So

$$
4 g\left(a x_{1}+c x_{3}\right)=4 a^{2} g\left(x_{1}\right)+4 c^{2} g\left(x_{3}\right)+2 g\left(a x_{1}+c x_{3}\right)-2 g\left(a x_{1}-c x_{3}\right) .
$$

Adding and subtracting $2 g\left(b x_{2}\right)$ to (3.7), we get

$$
\begin{aligned}
4 g\left(a x_{1}+c x_{3}\right)=4 a^{2} g\left(x_{1}\right)+4 c^{2} g\left(x_{3}\right)+g\left(a x_{1}\right. & \left.+c x_{3}+b x_{2}\right)+g\left(a x_{1}+c x_{3}-b x_{2}\right) \\
& -g\left(a x_{1}-c x_{3}+b x_{2}\right)-g\left(a x_{1}-c x_{3}-b x_{2}\right)
\end{aligned}
$$

for $x_{1}, x_{2}, x_{3} \in X$.

Adding $4 g\left(b x_{2}\right)$ to (3.8) on both sides, we obtain

$$
\begin{aligned}
4 g\left(a x_{1}+c x_{3}\right)+4 g\left(b x_{2}\right)=4 a^{2} g\left(x_{1}\right) & +4 c^{2} g\left(x_{3}\right)+g\left(a x_{1}+c x_{3}+b x_{2}\right)+g\left(a x_{1}+c x_{3}-b x_{2}\right) \\
& -g\left(a x_{1}-c x_{3}+b x_{2}\right)-g\left(a x_{1}-c x_{3}-b x_{2}\right)+4 g\left(b x_{2}\right)
\end{aligned}
$$

for $x_{1}, x_{2}, x_{3} \in X$. By (3.9) and (3.3), we get

$$
\begin{aligned}
4 g\left(a x_{1}+c x_{3}\right)+4 g\left(b x_{2}\right) & =4 a^{2} g\left(x_{1}\right)+4 c^{2} g\left(x_{3}\right)+4 b^{2} g\left(x_{2}\right)+g\left(a x_{1}+c x_{3}+b x_{2}\right) \\
+ & g\left(a x_{1}+c x_{3}-b x_{2}\right)-g\left(a x_{1}-c x_{3}+b x_{2}\right)-g\left(-a x_{1}+c x_{3}+b x_{2}\right)
\end{aligned}
$$


for $x_{1}, x_{2}, x_{3} \in X$. Using (3.10), (3.3) and the evenness of $g$, we get

$$
\begin{array}{r}
g\left(a x_{1}+b x_{2}+c x_{3}\right)+g\left(a x_{1}-b x_{2}+c x_{3}\right)-g\left(a x_{1}+b x_{2}-c x_{3}\right)-g\left(-a x_{1}+b x_{2}+c x_{3}\right) \\
+4 a^{2} g\left(x_{1}\right)+4 b^{2} g\left(x_{2}\right)+4 c^{2} g\left(x_{3}\right)=4 g\left(a x_{1}+c x_{3}\right)+4 g\left(b x_{2}\right)
\end{array}
$$

for all $x_{1}, x_{2}, x_{3} \in X$.

Conversely, assume that $g: X \rightarrow Y$ satisfies the functional equation (1.3).

Replacing $\left(x_{1}, x_{2}, x_{3}\right)$ by $\left(\frac{x}{a}, 0, \frac{y}{c}\right)$ in (1.3), we get

$$
g(x-y)-g(-x+y)+g(x+y)-g(x-y)+4 g(x)+4 g(y)=4 g(x+y)
$$

for all $x, y \in X$. Using (1.3) and the evenness of $g$, we get

$$
g(x+y)+g(x-y)=2 g(x)+2 g(y)
$$

which is quadratic.

\section{Stability results for (1.3): Odd case and direct method}

In this section, we present the Hyers-Ulam stability of the functional equation (1.3) for an odd mapping case.

Theorem 3. Let $j \in\{-1,1\}$ and $\alpha: X^{3} \rightarrow[0, \infty)$ be a function such that

$$
\sum_{k=0}^{\infty} \frac{\alpha\left(a^{k j} x_{1}, a^{k j} x_{2}, a^{k j} x_{3}\right)}{a^{k j}}<\infty
$$

for all $x_{1}, x_{2}, x_{3} \in X$. Let $g: X \rightarrow Y$ be an odd mapping satisfying the inequality

$$
\left\|D g\left(x_{1}, x_{2}, x_{3}\right)\right\| \leq \alpha\left(x_{1}, x_{2}, x_{3}\right)
$$

for all $x_{1}, x_{2}, x_{3} \in X$. There exists a unique additive mapping $A: X \rightarrow Y$ which satisfies the functional equation (1.3) and

$$
\|g(x)-A(x)\| \leq \frac{1}{2} \sum_{k=\frac{1-j}{2}}^{\infty} \frac{\alpha\left(a^{k j} x_{1}, 0,0\right)}{a^{k j}}
$$

for all $x_{1} \in X$. The mapping $A(x)$ is defined by,

$$
A(x)=\lim _{k \rightarrow \infty} \frac{g\left(a^{k j} x_{1}\right)}{a^{k j}} \text { for all } x \in X
$$

Proof. Assume that $j=1$. Replacing $\left(x_{1}, x_{2}, x_{3}\right)$ by $(x, 0,0)$ in (4.2) and using the oddness of $g$, we get

$$
\|2 g(a x)-2 a g(x)\| \leq \alpha(x, 0,0)
$$


for all $x \in X$. It follows from (4.3) that

$$
\left\|\frac{g(a x)}{a}-g(x)\right\| \leq \frac{1}{2 a} \alpha(x, 0,0)
$$

for all $x \in X$. Replacing $x$ by $a x$ in (4.4) and dividing by $a$, we obtain

$$
\left\|\frac{g\left(a^{2} x\right)}{a^{2}}-\frac{g(a x)}{a}\right\| \leq \frac{1}{2 a^{2}} \alpha(a x, 0,0)
$$

for all $x \in X$. It follows from (4.4) and (4.5) that

$$
\left\|\frac{g\left(a^{2} x\right)}{a^{2}}-g(x)\right\| \leq \frac{1}{2 a}\left[\alpha(x, 0,0)+\frac{\alpha(a x, 0,0)}{a}\right]
$$

for all $x \in X$. Similarly, for any positive integer $n$, we have

$$
\left\|g(x)-\frac{g\left(a^{n} x\right)}{a^{n}}\right\| \leq \frac{1}{2 a} \sum_{k=0}^{n-1} \frac{\alpha\left(a^{k} x, 0,0\right)}{a^{k}} \leq \frac{1}{2 a} \sum_{k=0}^{\infty} \frac{\alpha\left(a^{k} x, 0,0\right)}{a^{k}}
$$

for all $x \in X$. In order to prove convergence of the sequence $\left\{\frac{g\left(a^{k} x\right)}{a^{k}}\right\}$, replacing $x$ by $a^{m} x$ and dividing $a^{m}$ in (4.7) for any $m, n>0$, we get

$$
\begin{aligned}
\left\|\frac{g\left(a^{m} x\right)}{a^{m}}-\frac{g\left(a^{m+n} x\right)}{a^{m+n}}\right\| & =\frac{1}{2 a^{m}}\left\|g\left(a^{m} x\right)-\frac{g\left(a^{m} a^{n} x\right)}{a^{n}}\right\| \\
& \leq \frac{1}{2 a} \sum_{m=0}^{n-1} \frac{\alpha\left(a^{m+n} x, 0,0\right)}{a^{m+n}} \\
& \leq \frac{1}{2 a} \sum_{m=0}^{n-1} \frac{\alpha\left(a^{m+n} x, 0,0\right)}{a^{m+n}} \\
& \rightarrow 0 \text { as } m \rightarrow \infty .
\end{aligned}
$$

Hence the sequence $\left\{\frac{g\left(a^{n} x\right)}{a^{n}}\right\}$ is a Cauchy sequence. Since $Y$ is complete, there exists a mapping $A: X \rightarrow Y$ such that

$$
A(x)=\lim _{n \rightarrow \infty} \frac{g\left(a^{n} x\right)}{a^{n}}, \forall x \in X .
$$

Letting $n \rightarrow \infty$ in (4.8), we see that (4.8) holds for $x \in X$.

To prove that $A$ satisfies (1.3), replacing $\left(x_{1}, x_{2}, x_{3}\right)$ by $\left(a^{n} x, a^{n} x, a^{n} x\right)$ and dividing $a^{n}$ in (4.1), we obtain

$$
\frac{1}{a^{n}}\left\|D g\left(a^{n} x, a^{n} x, a^{n} x\right)\right\| \leq \frac{1}{a^{n}} \alpha\left(a^{n} x, a^{n} x, a^{n} x\right)
$$

for all $x_{1}, x_{2}, x_{3} \in X$. Letting $m \rightarrow \infty$ in the above inequality and using the definition of $A(x)$, we see that $D A\left(x_{1}, x_{2}, x_{3}\right)=0$. Hence $A$ satisfies (1.3) for all $x_{1}, x_{2}, x_{3} \in X$.

To show that $A$ is unique, let $B(x)$ be another additive mapping satisfying (4.2). Then

$$
\begin{aligned}
\|A(x)-B(x)\| & =\frac{1}{a^{n}}\left\|A\left(a^{n} x\right)-B\left(a^{n} x\right)\right\| \\
& \leq \frac{1}{a^{n}}\left\{\left\|A\left(a^{n} x\right)-g\left(a^{n} x\right)\right\|+\left\|g\left(a^{n} x\right)-B\left(a^{n} x\right)\right\|\right\} \\
& \rightarrow 0 \text { as } n \rightarrow \infty .
\end{aligned}
$$


Hence $A$ is unique.

Assume that $j=-1$. Replacing $x$ by $\frac{x}{a}$ in (4.3), we get

$$
\left\|a g(x)-a^{2} g\left(\frac{x}{a}\right)\right\| \leq \alpha\left(\frac{x}{a}, 0,0\right)
$$

for all $x \in X$. The rest of the proof is similar to the proof of the case $j=1$. This completes the proof of the theorem.

The following corollary is an immediate consequence of Theorem 3 concerning the stability of (1.3).

Corollary 1. Let $\epsilon$ and $p$ be nonnegative real numbers. Let $g: X \rightarrow Y$ be an odd mapping satisfiying the inequality

$$
\begin{aligned}
& \text { \|Dg( } \left.x_{1}, x_{2}, x_{3}\right) \|
\end{aligned}
$$

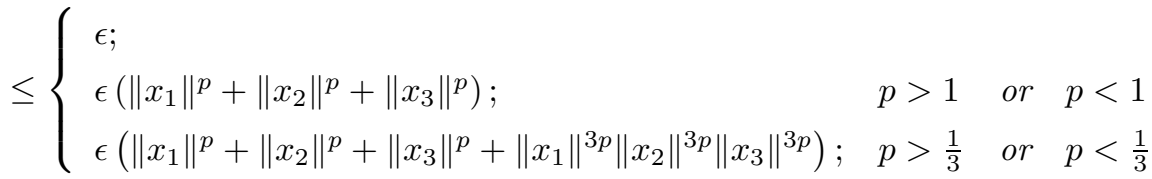

for all $x_{1}, x_{2}, x_{3} \in X$. Then there exists a unique additive mapping $A: X \rightarrow Y$ such that

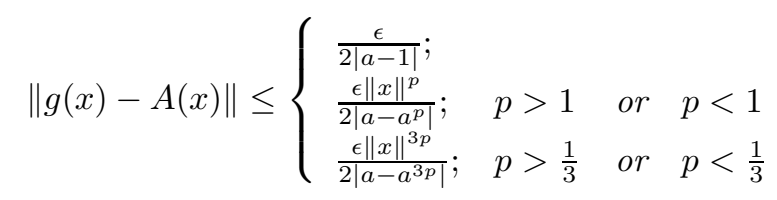

for all $x \in X$.

Proof. Letting

$$
\alpha\left(x_{1}, x_{2}, x_{3}\right)=\left\{\begin{array}{l}
\epsilon ; \\
\epsilon\left(\left\|x_{1}\right\|^{p}+\left\|x_{2}\right\|^{p}+\left\|x_{3}\right\|^{p}\right) ; \\
\epsilon\left(\left\|x_{1}\right\|^{p}+\left\|x_{2}\right\|^{p}+\left\|x_{3}\right\|^{p}+\left\|x_{1}\right\|^{3 p}\left\|x_{2}\right\|^{3 p}\left\|x_{3}\right\|^{3 p}\right)
\end{array}\right.
$$

for all $x_{1}, x_{2}, x_{3} \in X$, we can get the result.

\section{Stability results for (1.3): Even case and direct method}

In this section, we discuss the Hyers-Ulam stability of the functional equation (1.3) for an even mapping case by using the direct method. 
Theorem 4. Let $j \in\{-1,1\}$ and $\alpha: X^{3} \rightarrow[0, \infty)$ be a function such that

$$
\sum_{k=0}^{\infty} \frac{\alpha\left(a^{k j} x_{1}, a^{k j} x_{2}, a^{k j} x_{3}\right)}{a^{k j}}<\infty
$$

for all $x_{1}, x_{2}, x_{3} \in X$. Let $g: X \rightarrow Y$ be an even mapping satisfying $g(0)=0$ and the inequality

$$
\left\|D g\left(x_{1}, x_{2}, x_{3}\right)\right\| \leq \alpha\left(x_{1}, x_{2}, x_{3}\right)
$$

for all $x_{1}, x_{2}, x_{3} \in X$. There exists a unique additive mapping $Q: X \rightarrow Y$ which satisfies the functional equation (1.3) and

$$
\|g(x)-Q(x)\| \leq \frac{1}{4 a^{2}} \sum_{k=\frac{1-j}{2}}^{\infty} \frac{\alpha\left(a^{k j} x, 0,0\right)}{a^{2 k j}}
$$

for all $x \in X$. The mapping $Q(x)$ is defined by

$$
Q(x)=\lim _{n \rightarrow \infty} \frac{g\left(a^{k j} x\right)}{a^{2 k j}}
$$

for all $x \in X$.

Proof. Assume that $j=1$. Replacing $\left(x_{1}, x_{2}, x_{3}\right)$ by $(x, 0,0)$ in (5.2), we get

$$
\left\|4 g(a x)-4 a^{2} g(x)\right\| \leq \alpha(x, 0,0)
$$

for all $x \in X$. It follows from (5.5) that

$$
\left\|\frac{g(a x)}{a^{2}}-g(x)\right\| \leq \frac{1}{4 a^{2}} \alpha(x, 0,0)
$$

for all $x \in X$. Replacing $x$ by ax in (5.6) and dividing by $a^{2}$, we obtain

$$
\left\|\frac{g\left(a^{2} x\right)}{a^{4}}-\frac{g(a x)}{a^{2}}\right\| \leq \frac{1}{4 a^{4}} \alpha(a x, 0,0)
$$

for all $x \in X$. It follows from (5.6) and (5.7) that

$$
\left\|\frac{g\left(a^{2} x\right)}{a^{4}}-g(x)\right\| \leq \frac{1}{4 a^{2}}\left[\alpha(x, 0,0)+\frac{\alpha(a x, 0,0)}{a^{2}}\right]
$$

for all $x \in X$. Inductively, we have

$$
\left\|g(x)-\frac{g\left(a^{n} x\right)}{a^{2 n}}\right\| \leq \frac{1}{4 a^{2}} \sum_{k=0}^{n-1} \frac{\alpha\left(a^{k} x, 0,0\right)}{a^{2 k}} \leq \frac{1}{a^{3}} \sum_{k=0}^{\infty} \frac{\alpha\left(a^{k} x, 0,0\right)}{a^{2 k}}
$$


for all $x \in X$. In order to prove convergence of the sequence $\left\{\frac{g\left(a^{k} x\right)}{a^{2 k}}\right\}$, replacing $x$ by $a^{m} x$ and dividing $a^{m}$ in (5.9) for any $m, n>0$, we get

$$
\begin{aligned}
\left\|\frac{g\left(a^{m} x\right)}{a^{2 m}}-\frac{g\left(a^{m+n} x\right)}{a^{2(m+n)}}\right\| & =\frac{1}{a^{2 m}}\left\|g\left(a^{m} x\right)-\frac{g\left(a^{m} a^{n} x\right)}{a^{2 n}}\right\| \\
& \leq \frac{1}{a^{3}} \sum_{m=0}^{n-1} \frac{\alpha\left(a^{m+n} x, 0,0\right)}{a^{2(m+n)}} \\
& \leq \frac{1}{a^{3}} \sum_{m=0}^{n-1} \frac{\alpha\left(a^{m+n} x, 0,0\right)}{a^{2(m+n)}} \\
& \rightarrow 0 \text { as } m \rightarrow \infty .
\end{aligned}
$$

Hence the sequence $\left\{\frac{g\left(a^{n} x\right)}{a^{2 n}}\right\}$ is a Cauchy sequence. Since $Y$ is complete, there exists a mapping $Q: X \rightarrow Y$ such that

$$
Q(x)=\lim _{n \rightarrow \infty} \frac{g\left(a^{n} x\right)}{a^{2 n}}, \forall x \in X .
$$

Letting $n \rightarrow \infty$ in (5.10) we see that (5.10) holds for $x \in X$.

To prove that $Q$ satisfies (1.3), replacing $\left(x_{1}, x_{2}, x_{3}\right)$ by $\left(a^{n} x, a^{n} x, a^{n} x\right)$ and dividing $a^{2 n}$ in (5.2), we obtain

$$
\frac{1}{a^{2 n}}\left\|D g\left(a^{n} x, a^{n} x, a^{n} x\right)\right\| \leq \frac{1}{a^{2 n}} \alpha\left(a^{n} x, a^{n} x, a^{n} x\right)
$$

for all $x_{1}, x_{2}, x_{3} \in X$. Letting $n \rightarrow \infty$ in the above inequality and using the definition of $Q(x)$, we see that $D Q\left(x_{1}, x_{2}, x_{3}\right)=0$. Hence $Q$ satisfies (1.3) for all $x_{1}, x_{2}, x_{3} \in X$.

To show that $Q$ is unique, let $B(x)$ be another quadratic mapping satisfying (5.4). Then

$$
\begin{aligned}
\|Q(x)-B(x)\| & =\frac{1}{a^{2 n}}\left\|Q\left(a^{n} x\right)-B\left(a^{n} x\right)\right\| \\
& \leq \frac{1}{a^{2 n}}\left\{\left\|Q\left(a^{n} x\right)-g\left(a^{n} x\right)\right\|+\left\|g\left(a^{n} x\right)-B\left(a^{n} x\right)\right\|\right\} \\
& \rightarrow 0 \text { as } n \rightarrow \infty .
\end{aligned}
$$

Hence $Q$ is unique.

Assume that $j=-1$. Replacing $x$ by $\frac{x}{a}$ in (5.5), we get

$$
\left\|a g(x)-a^{2} g\left(\frac{x}{a}\right)\right\| \leq \frac{1}{4} \alpha\left(\frac{x}{a}, 0,0\right)
$$

for all $x \in X$. The rest of the proof is similar to the proof of the case $j=1$. This completes the proof of the theorem.

The following corollary is an immediate consequence of Theorem 4 concerning the stability of (1.3). 
Corollary 2. Let $\epsilon$ and $p$ be nonnegative real numbers. Let $g_{q}: X \rightarrow Y$ be an even mapping satisfiying $g(0)=0$ and the inequality

$$
\begin{aligned}
& \text { \|Dg( } \left.x_{1}, x_{2}, x_{3}\right) \| \\
& \leq\left\{\begin{array}{lll}
\epsilon & & \\
\epsilon\left(\left\|x_{1}\right\|^{p}+\left\|x_{2}\right\|^{p}+\left\|x_{3}\right\|^{p}\right) ; & p>2 & \text { or } p<2 \\
\epsilon\left(\left\|x_{1}\right\|^{p}\left\|x_{2}\right\|^{p}\left\|x_{3}\right\|^{p}+\left\{\left\|x_{1}\right\|^{3 p}\left\|x_{2}\right\|^{3 p}\left\|x_{3}\right\|^{3 p}\right\}\right) ; & p>\frac{2}{3} & \text { or } \quad p<\frac{2}{3}
\end{array}\right.
\end{aligned}
$$

for all $x_{1}, x_{2}, x_{3} \in X$. Then there exists a unique quadratic mapping $Q: X \rightarrow Y$ such that

$$
\|g(x)-Q(x)\| \leq\left\{\begin{array}{l}
\frac{\epsilon}{4\left|a^{2}-1\right|} \\
\frac{\epsilon\|x\|^{p}}{4\left|a^{2}-a^{p}\right|} \\
\frac{\epsilon\|x\|^{3 p}}{4\left|a^{2}-a^{3 p}\right|}
\end{array}\right.
$$

for all $x \in X$.

Proof. Letting

$$
\alpha\left(x_{1}, x_{2}, x_{3}\right)=\left\{\begin{array}{l}
\epsilon \\
\epsilon\left(\left\|x_{1}\right\|^{p}+\left\|x_{2}\right\|^{p}+\left\|x_{3}\right\|^{p}\right) ; \\
\epsilon\left(\left\|x_{1}\right\|^{p}\left\|x_{2}\right\|^{p}\left\|x_{3}\right\|^{p}+\left\{\left\|x_{1}\right\|^{3 p}\left\|x_{2}\right\|^{3 p}\left\|x_{3}\right\|^{3 p}\right\}\right) ;
\end{array}\right.
$$

for all $x_{1}, x_{2}, x_{3} \in X$, we get the result.

\section{Stability results of (1.3): Mixed case}

In this section, we establish the Hyers-Ulam stability of the functional equation(1.3) for a mixed mapping case.

Theorem 5. Let $j \in\{-1,1\}$ and $\alpha: X^{3} \rightarrow[0, \infty)$ be a function satisfying (1.3) for all $x_{1}, x_{2}, x_{3} \in$ $X$. Let $g: X \rightarrow Y$ be a mapping satisfying the inequality

$$
\left\|D g\left(x_{1}, x_{2}, x_{3}\right)\right\| \leq \alpha\left(x_{1}, x_{2}, x_{3}\right)
$$

for all $x_{1}, x_{2}, x_{3} \in X$. There exist a unique additive mapping $A: X \rightarrow Y$ and a unique quadratic mapping $Q: X \rightarrow Y$ which satisfies the functional equation (1.3) and

$$
\begin{aligned}
\|f(x)-A(x)-Q(x)\| \leq & \frac{1}{2}\left\{\left[\frac{1}{2 a} \sum_{k=\frac{1-j}{2}}^{\infty}\left[\frac{\alpha\left(a^{k j} x, 0,0\right)}{a^{k j}}+\frac{\alpha\left(-a^{k j} x, 0,0\right)}{a^{k j}}\right]\right]\right. \\
& \left.+\frac{1}{4 n^{2}}\left[\sum_{k=\frac{1-j}{2}}^{\infty}\left[\frac{\alpha\left(a^{k j} x, 0,0\right)}{a^{2 k j}}+\frac{\alpha\left(-a^{k j} x, 0,0\right)}{a^{2 k j}}\right]\right]\right\}
\end{aligned}
$$

for all $x \in X$. The mapping $A(x)$ and $Q(x)$ are defined in (4.2) and (5.10), respectively. 
Proof. Let $g_{o}(x)=\frac{g_{a}(x)-g_{a}(-x)}{2}$ for all $x \in X$. Then $g_{o}(0)=0$ and $g_{o}(-x)=-g_{o}(x)$ for all $x \in X$.

Hence

$$
\begin{aligned}
\left\|D g_{o}\left(x_{1}, x_{2}, x_{3}\right)\right\| & \leq \frac{1}{2}\left\{\left\|D g_{a}\left(x_{1}, x_{2}, x_{3}\right)\right\|+\left\|D g_{a}\left(-x_{1},-x_{2},-x_{3}\right)\right\|\right\} \\
& \leq \frac{\alpha\left(x_{1}, x_{2}, x_{3}\right)}{2}+\frac{\alpha\left(-x_{1},-x_{2},-x_{3}\right)}{2}
\end{aligned}
$$

for all $x_{1}, x_{2}, x_{3} \in X$. By Theorem 3 we have

$$
\left\|g_{o}(x)-A(x)\right\| \leq \frac{1}{4 a} \sum_{k=\frac{1-j}{2}}^{\infty}\left[\frac{\alpha\left(a^{k j} x, 0,0\right)}{a^{k j}}+\frac{\alpha\left(-a^{k j} x, 0,0\right)}{a^{k j}}\right]
$$

for all $x \in X$.

Let $g_{e}(x)=\frac{g_{q}(x)+g_{q}(-x)}{2}$ for all $x \in X$. Then $g_{e}(0)=0$ and $g_{e}(-x)=g_{e}(x)$ for all $x \in X$. Hence,

$$
\begin{aligned}
\left\|D g_{e}\left(x_{1}, x_{2}, x_{3}\right)\right\| & \leq \frac{1}{2}\left\{\left\|D g_{q}\left(x_{1}, x_{2}, x_{3}\right)\right\|+\left\|D g_{q}\left(-x_{1},-x_{2},-x_{3}\right)\right\|\right\} \\
& \leq \frac{\alpha\left(x_{1}, x_{2}, x_{3}\right)}{2}+\frac{\alpha\left(-x_{1},-x_{2},-x_{3}\right)}{2}
\end{aligned}
$$

for all $x_{1}, x_{2}, x_{3} \in X$.

By Theorem 4, we have

$$
\left\|g_{e}(x)-Q(x)\right\| \leq \frac{1}{8 a^{2}} \sum_{k=\frac{1-j}{2}}^{\infty}\left[\frac{\alpha\left(a^{k j} x, 0,0\right)}{a^{2 k j}}+\frac{\alpha\left(-a^{k j} x, 0,0\right)}{a^{2 k j}}\right]
$$

for all $x \in X$. Then

$$
g(x)=g_{e}(x)+g_{o}(-x)
$$

for all $x \in X$. It follows from (6.2), (6.3) and (6.4) that

$$
\begin{aligned}
\|g(x)-A(x)-Q(x)\|= & \left\|g_{e}(x)+g_{o}(-x)-A(x)-Q(x)\right\| \\
\leq & \left\|g_{o}(-x)-A(x)\right\|+\left\|g_{e}(x)-Q(x)\right\| \\
\leq & \frac{1}{4 a} \sum_{k=\frac{1-j}{2}}^{\infty}\left[\frac{\alpha\left(a^{k j} x, 0,0\right)}{a^{k j}}+\frac{\alpha\left(-a^{k j} x, 0,0\right)}{a^{k j}}\right] \\
& +\frac{1}{8 a^{2}} \sum_{k=\frac{1-j}{2}}^{\infty}\left[\frac{\alpha\left(a^{k j} x, 0,0\right)}{a^{2 k j}}+\frac{\alpha\left(-a^{k j} x, 0,0\right)}{a^{2 k j}}\right]
\end{aligned}
$$

for all $x \in X$. Hence the theorem is proved.

Using Corollaries 1 and 2, we have the following corollary concerning the stability of (1.3). 
Corollary 3. Let $\lambda$ and $s$ be a nonnegative real numbers. Let $g_{q}: X \rightarrow Y$ be a mapping satisfiying the inequality

$$
\left\|D_{g}\left(x_{1}, x_{2}, x_{3}\right)\right\| \leq \begin{cases}\lambda ; & s \neq 1,2 \\ \lambda\left(\left\|x_{1}\right\|^{s}+\left\|x_{2}\right\|^{s}+\left\|x_{3}\right\|^{s}\right) ; & \\ \lambda\left(\left\|x_{1}\right\|^{s}+\left\|x_{2}\right\|^{s}+\left\|x_{3}\right\|^{s}\right)+\left\{\left\|x_{1}\right\|^{3 s}+\left\|x_{2}\right\|^{3 s}+\left\|x_{3}\right\|^{3 s}\right\} ; & s \neq \frac{1}{3}, \frac{2}{3}\end{cases}
$$

for all $x_{1}, x_{2}, x_{3} \in X$. Then there exist a unique additive function $A: X \rightarrow Y$ and a unique quadratic mapping $Q: X \rightarrow Y$ such that

$$
\|g(x)-A(x)-Q(x)\| \leq\left\{\begin{array}{l}
\frac{\lambda}{2}\left[\frac{1}{|a-1|}+\frac{1}{2\left|a^{2}-1\right|}\right] \\
\frac{\left.\lambda\|x\|\right|^{S}}{2}\left[\frac{1}{\left|a-a^{S}\right|}+\frac{1}{2\left|a^{2}-a^{S}\right|}\right] \\
\frac{\lambda\|x\|^{3 S}}{2}\left[\frac{1}{\left|a-a^{3 S}\right|}+\frac{1}{2\left|a^{2}-a^{3 S}\right|}\right]
\end{array}\right.
$$

for all $x \in X$.

\section{$7 \quad$ Fixed point stability of (1.3): Odd mapping case}

The following theorems are useful to prove our fixed point stability results.

Theorem 6. [12] (Banach Contraction Principle) Let $(X, d)$ be a complete metric space and consider a mapping $T: X \rightarrow X$ which is strictly contractive mapping.

(A1) $d(T x, T y) \leq L d(x, y)$ for some (Lipschitz constant) $L<1$.

(i) The mapping $T$ has one and only fixed point $x^{*}=T\left(x^{*}\right)$;

(ii) The fixed point for each given element $x^{*}$ is globally contractive, that is,

(A2) $\lim _{n \rightarrow \infty} T^{n} x=x^{*}$ for any starting point $x \in X$;

(iii) One has the following estimation inequalities

(A3) $d\left(T^{n} x, x^{*}\right) \leq \frac{1}{1-L} d\left(T^{n} x, T^{n+1} x\right), \forall n \geq 0, \forall x \in X$;

(A4) $d\left(x, x^{*}\right)=\frac{1}{1-L} d\left(x, x^{*}\right), \forall x \in X$.

Theorem 7. [12] (Alternative Fixed Point Theorem) Suppose that for a complete generalized metric space $(X, d)$ and a strictly contractive mapping $T: X \rightarrow X$ with Lipschitz constant $L$. Then for each given element $x \in X$,

(B1) $d\left(T^{n} x, T^{n+1} x\right)=\infty, \forall n \geq 0$;

(B2) there exists a natural number $n_{0}$ such that 
(i) $d\left(T^{n} x, T^{n+1} x\right)<\infty, \forall n \geq 0$;

(ii) The sequence $\left\{T^{n} x\right\}$ is convergent to a fixed point $y^{*}$ of $T$;

(iii) $y^{*}$ is the unique fixed point of $T$ in the set $Y=\left\{y \in Y: d\left(T^{n_{0}}, y\right)<\infty\right\}$;

(iv) $d\left(y^{*}, y\right) \leq \frac{1}{1-L} d(y, T y)$ for all $y \in Y$.

In this method, we investigate the Hyers-Ulam stability of the functional equation (1.3) for an odd mapping case by using fixed point method.

Theorem 8. Let $g: W \rightarrow B$ be an odd mapping for which there exists a function $\alpha: W^{3} \rightarrow[0, \infty)$ with the condition

$$
\lim _{n \rightarrow \infty} \frac{\alpha\left(a_{i}^{k} x_{1}, a_{i}^{k} x_{2}, a_{i}^{k} x_{3}\right)}{a_{i}^{k}}=0
$$

for $a_{i}=\left\{\begin{array}{ll}a & i=0 \\ \frac{1}{a} & i=1,\end{array}\right.$ such that the functional inequality

$$
\left\|D g\left(x_{1}, x_{2}, x_{3}\right)\right\| \leq \alpha\left(x_{1}, x_{2}, x_{3}\right)
$$

for all $x_{1}, x_{2}, x_{3} \in W$. If there exists $L=L(i)$ such that the function $x \rightarrow \beta(x)=\frac{1}{2} \alpha\left(\frac{x}{a}, 0,0\right)$ has the property

$$
\frac{1}{a_{i}} \beta\left(a_{i} x\right)=L(\beta(x))
$$

for all $x \in W$. Then there exists a unique additive function $A: W \rightarrow B$ satisfying the functional equation (1.3) and

$$
\|g(x)-A(x)\| \leq \frac{L^{1-i}}{1-L} \beta(x)
$$

for all $x \in W$.

Proof. Consider the set $X=\{P \mid P: W \rightarrow B, P(0)=0\}$ and introduce the generalized metric on $X$.

$$
d(p, q)=\inf \{k \in(0, \infty):\|p(x)-q(x)\| \leq \beta(x), x \in W\}
$$

It is easy to see that $(X, d)$ is complete.

Define $T: X \rightarrow X$ by $T_{p}(x)=\frac{1}{a_{i}} p\left(a_{i} x\right)$ for all $x \in W$. Now $p, q \in X$,

$$
\begin{aligned}
& d(p, q) \leq k \\
& \Rightarrow\|p(x)-q(x)\| \leq k \beta(x), x \in W . \\
& \Rightarrow\left\|\frac{1}{a_{i}} p\left(a_{i} x\right)-\frac{1}{a_{i}} q\left(a_{i} x\right)\right\| \leq \frac{1}{a_{i}} k \beta\left(a_{i} x\right), \forall x \in W \\
& \Rightarrow\left\|T_{p}(x)-T_{q}(x)\right\| \leq L k \beta(x), \forall x \in W \\
& \Rightarrow d\left(T_{p}, T_{q}\right) \leq L k .
\end{aligned}
$$


This implies $d\left(T_{p}, T_{q}\right) \leq L d(p, q)$ for all $p, q \in X$. That is, $T$ is a strictly contractive mapping on $X$ with Lipschitz constant $L$. It follows from (4.3) that

$$
\|2 g(a x)-2 a g(x)\| \leq \alpha(x, 0,0)
$$

for all $x \in W$. It follows from (7.5) that,

$$
\left\|g(x)-\frac{g(a x)}{a}\right\| \leq \frac{1}{2 a} \alpha(x, 0,0)
$$

for all $x \in W$. Using (6.2), for this case $i=0$, it reduces to

$$
\left\|g(x)-\frac{g(a x)}{a}\right\| \leq \frac{1}{a} \beta(x)
$$

for all $x \in W$. Thus

$$
d\left(g_{a}, T g_{a}\right) \leq \frac{1}{a}=L=L^{1}<\infty .
$$

Again replacing $x$ by $\frac{x}{a}$ in (7.5), we get

$$
\left\|g(x)-a g\left(\frac{x}{a}\right)\right\| \leq \frac{1}{2} \alpha\left(\frac{x}{a}, 0,0\right)
$$

for all $x \in W$.

By using (7.3) for the case $i=1$, it reduces to

$$
\left\|g(x)-a g\left(\frac{x}{a}\right)\right\| \leq \beta(x) .
$$

That is, $d(g, T g) \leq 1 \Rightarrow d(g, T g) \leq 1=L^{0}<\infty$. In the above case, we have $d(g, T g) \leq L^{1-i}$. Therefore $\left(B_{2}(i)\right)$ holds. From $\left(B_{2}(i i)\right)$, it follows that there exists a fixed point $A$ of $T$ in $X$ such that

$$
A(x)=\lim _{i \rightarrow \infty} \frac{g_{a}\left(a_{i}^{k} x\right)}{a_{i}^{k}}, \forall x \in W .
$$

In order to prove $A: W \rightarrow B$ is additive, replacing $\left(x_{1}, x_{2}, x_{3}\right)$ by $\left(a_{i}^{k} x_{1}, a_{i}^{k} x_{2}, a_{i}^{k} x_{3}\right)$ in (7.2) and dividing $a_{i}^{k}$, it follows from (7.3) and (7.10) that $A$ satisfies (1.3) for all $x_{1}, x_{2}, x_{3} \in W$. By $\left(B_{2}(i i i)\right), A$ is the unique fixed point of $T$ in the set, $Y=\{g \in X: d(T g, A)<\infty\}$.

Using the fixed point alternative result, $A$ is the unique function such that

$$
\|g(x)-A(x)\| \leq k \beta(x)
$$

for all $x \in W$ and $k>0$. Finally, by $\left(B_{2}(i v)\right)$, we obtain

$$
d(g, A) \leq \frac{1}{1-L} d(g, T g) .
$$

That is, $d(g, A) \leq \frac{L^{1-i}}{1-L}$. Hence we conclude that

$$
\|g(x)-A(x)\| \leq \frac{L^{1-i}}{1-L} \beta(x)
$$

for all $x \in W$. This completes the proof of the theorem. 
Corollary 4. Let $g: W \rightarrow B$ be an odd mapping and assume that there exist real numbers $\lambda$ and s such that

$$
\left\|D_{g_{a}}\left(x_{1}, x_{2}, x_{3}\right)\right\| \leq\left\{\begin{array}{l}
\lambda \\
\lambda\left(\left\|x_{1}\right\|^{s}+\left\|x_{2}\right\|^{s}+\left\|x_{3}\right\|^{s}\right) \\
\lambda\left(\left\|x_{1}\right\|^{s}+\left\|x_{2}\right\|^{s}+\left\|x_{3}\right\|^{s}\right)+\left\{\left\|x_{1}\right\|^{3 s}+\left\|x_{2}\right\|^{3 s}+\left\|x_{3}\right\|^{3 s}\right\}
\end{array}\right.
$$

for all $x_{1}, x_{2}, x_{3} \in X$. Then there exists a unique additive mapping $A: W \rightarrow B$ such that

$$
\|g(x)-A(x)\| \leq \begin{cases}\frac{\lambda}{2|a-1|} ; & \\ \frac{\lambda\|x\|^{s}}{2 \mid a-a^{s}} ; & s \neq 1 \\ \frac{\lambda\|x\|^{s}}{2\left|a-a^{3 s}\right|} ; & s \neq \frac{1}{3}\end{cases}
$$

for all $x \in X$.

Proof. Let

$$
\alpha\left(x_{1}, x_{2}, x_{3}\right)=\left\{\begin{array}{l}
\lambda \\
\lambda\left(\left\|x_{1}\right\|^{S}+\left\|x_{2}\right\|^{S}+\left\|x_{3}\right\|^{S}\right) ; \\
\lambda\left(\left\|x_{1}\right\|^{S}+\left\|x_{2}\right\|^{S}+\left\|x_{3}\right\|^{S}\right)+\left\{\lambda\left(\left\|x_{1}\right\|^{3 S}+\left\|x_{2}\right\|^{3 S}+\left\|x_{3}\right\|^{3 S}\right)\right\}
\end{array}\right.
$$

for all $x_{1}, x_{2}, x_{3} \in W$.

Now,

$$
\begin{aligned}
& \frac{\alpha\left(a_{i}^{k} x_{1}, a_{i}^{k} x_{2}, a_{i}^{k} x_{3}\right)}{a_{i}^{k}} \\
& =\left\{\begin{array}{l}
\frac{\lambda}{a_{i}^{k}} ; \\
\frac{\lambda}{a_{i}^{k}}\left(\left\|a_{i}^{k} x_{1}\right\|^{S}+\left\|a_{i}^{k} x_{2}\right\|^{S}+\left\|a_{i}^{k} x_{3}\right\|^{S}\right) ; \\
\frac{\lambda}{a_{i}^{k}}\left(\left\|a_{i}^{k} x_{1}\right\|^{S}+\left\|a_{i}^{k} x_{2}\right\|^{S}+\left\|a_{i}^{k} x_{3}\right\|^{S}\right)+\left\{\left\|a_{i}^{k} x_{1}\right\|^{3 S}+\left\|a_{i}^{k} x_{2}\right\|^{3 S}+\left\|a_{i}^{k} x_{3}\right\|^{3 S}\right\}
\end{array}\right. \\
& =\left\{\begin{array}{lll}
\rightarrow 0 & \text { as } & k \rightarrow \infty \\
\rightarrow 0 & \text { as } & k \rightarrow \infty \\
\rightarrow 0 & \text { as } & k \rightarrow \infty .
\end{array}\right.
\end{aligned}
$$

That is, (7.1) holds. But we have $\beta(x)=\frac{1}{2} \alpha\left(\frac{x}{a}, 0,0\right)$. Hence

$$
\beta(x)=\frac{1}{2} \alpha\left(\frac{x}{a}, 0,0\right)=\left\{\begin{array}{l}
\frac{\lambda}{2} \\
\frac{\lambda}{2 a^{S}}\left(\|x\|^{S}\right) \\
\frac{\lambda}{2 a^{S}}\left(\|x\|^{S}\right) .
\end{array}\right.
$$


Also

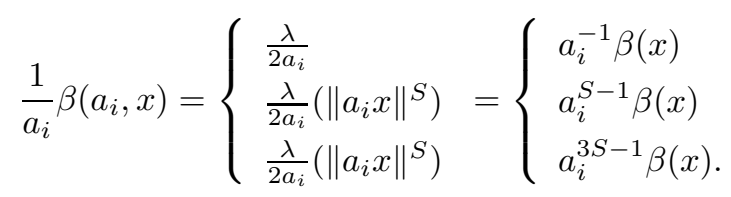

Hence the inequality (7.7) holds. Either $L=a^{-1}$ for $s=0$ if $i=0$ and $L=\frac{1}{a^{-1}}$ for $s=0$ if $i=1$.

Either $L=a^{s-1}$ for $s<1$ if $i=0$ and $L=\frac{1}{a^{s-1}}$ for $s>1$ if $i=1$.

Either $L=a^{3 s-1}$ for $s<1$ if $i=0$ and $L=\frac{1}{a^{3 s-1}}$ for $s>1$ if $i=1$.

Now from (17.2), we prove the following cases:

Case: $1 L=a^{-1}, i=0$

$$
\left\|g_{a}(x)-A(x)\right\| \leq \frac{L^{1-i}}{1-L} \beta(x)=\frac{\left(a^{-1}\right)^{1-0}}{1-a^{-1}} \frac{\lambda}{2}=\frac{\lambda}{2(a-1)} .
$$

Case: $2 L=\left(\frac{1}{a}\right)^{-1}, i=1$

$$
\left\|g_{a}(x)-A(x)\right\| \leq \frac{L^{1-i}}{1-L} \beta(x)=\frac{(a)^{1-1}}{1-a} \frac{\lambda}{2}=\frac{\lambda}{2(1-a)} .
$$

Case: $3 L=a^{s-1}, s<1, i=0$

$$
\left\|g_{a}(x)-A(x)\right\| \leq \frac{L^{1-i}}{1-L} \beta(x)=\frac{\left(a^{s-1}\right)^{1-0}}{1-a^{S-1}} \frac{\lambda}{2 a^{S}}\|x\|^{S}=\frac{\lambda\|x\|^{S}}{2\left|a-a^{S}\right|} .
$$

Case: $4 L=\left(\frac{1}{a}\right)^{S-1}, S>1, i=1$

$$
\left\|g_{a}(x)-A(x)\right\| \leq \frac{L^{1-i}}{1-L} \beta(x)=\frac{\left(a^{1-s}\right)^{1-1}}{1-a^{1-S}} \frac{\lambda}{2 a^{S}}\|x\|^{S}=\frac{\lambda\|x\|^{S}}{2\left(a^{S}-a\right)} .
$$

Case: $5 L=a^{3 s-1}, S<\frac{1}{3}, i=0$

$$
\left\|g_{a}(x)-A(x)\right\| \leq \frac{L^{1-i}}{1-L} \beta(x)=\frac{\left(a^{3 S-1}\right)^{1-0}}{1-a^{3 S-1}} \frac{\lambda}{2 a^{3 S}}\|x\|^{S}=\frac{\lambda\|x\|^{S}}{2\left(a-a^{3 S}\right)} .
$$

Case: $6 L=\left(\frac{1}{a}\right)^{-1}, i=1$

$$
\left\|g_{a}(x)-A(x)\right\| \leq \frac{L^{1-i}}{1-L} \beta(x)=\frac{\left(a^{1-3 S}\right)^{1-1}}{1-a^{1-3 S}} \frac{\lambda}{2 a^{3 S}}\|x\|^{S}=\frac{\lambda\|x\|^{S}}{2\left(a^{3 S}-a\right)} .
$$

Hence the proof of the corollary is completed.

\section{Fixed point stability of (1.3): Even mapping case}

In this method, we investigate the Hyers-Ulam stability of the functional equation (1.3) for an even case mapping by using fixed point method. 
Theorem 9. Let $g: W \rightarrow B$ be an even mapping for which there exists a function $\alpha: W^{3} \rightarrow[0, \infty)$ with the condition

$$
\lim _{n \rightarrow \infty} \frac{\alpha\left(a_{i}^{k} x_{1}, a_{i}^{k} x_{2}, a_{i}^{k} x_{3}\right)}{a_{i}^{2 k}}=0
$$

for $a_{i}=\left\{\begin{array}{ll}a & i=0 \\ \frac{1}{a} & i=1,\end{array}\right.$ such that the functional inequality

$$
\left\|D g\left(x_{1}, x_{2}, x_{3}\right)\right\| \leq \alpha\left(x_{1}, x_{2}, x_{3}\right)
$$

for all $x_{1}, x_{2}, x_{3} \in W$. If there exists $L=L(i)$ such that the function

$$
x \rightarrow \beta(x)=\frac{1}{2} \alpha\left(\frac{x}{a}, 0,0\right)
$$

has the property

$$
\frac{1}{a_{i}^{2}} \beta\left(a_{i} x\right)=L(\beta(x))
$$

for all $x \in W$, then there exists a unique quadratic mapping $Q: W \rightarrow B$ satisfying the functional equation (1.3) and

$$
\|g(x)-Q(x)\| \leq \frac{L^{1-i}}{1-L} \beta(x)
$$

for all $x \in W$.

Proof. Consider the set $X=\{P \mid P: W \rightarrow B, P(0)=0\}$ and introduce the generalized metric on $X$.

$$
d(p, q)=\inf \{k \in(0, \infty):\|p(x)-q(x)\| \leq \beta(x), x \in W\}
$$

It is easy to see that $(X, d)$ is complete.

Define $T: X \rightarrow X$ by $T_{p}(x)=\frac{1}{a_{i}^{2}} p\left(a_{i} x\right)$ for all $x \in W$. Now $p, q \in X$,

$$
\begin{aligned}
& d(p, q) \leq k \\
& \Rightarrow\|p(x)-q(x)\| \leq k \beta(x), x \in W . \\
& \Rightarrow\left\|\frac{1}{a_{i}^{2}} p\left(a_{i} x\right)-\frac{1}{a_{i}^{2}} q\left(a_{i} x\right)\right\| \leq \frac{1}{a_{i}^{2}} k \beta\left(a_{i} x\right), \forall x \in W \\
& \Rightarrow\left\|T_{p}(x)-T_{q}(x)\right\| \leq L k \beta(x), \forall x \in W \\
& \Rightarrow d\left(T_{p}, T_{q}\right) \leq L k .
\end{aligned}
$$

This implies $d\left(T_{p}, T_{q}\right) \leq L d(p, q)$ for all $p, q \in X$. That is, $T$ is a strictly contractive mapping on $X$ with Lipschitz constant $L$.

Replacing $\left(x_{1}, x_{2}, x_{3}\right)$ by $(x, 0,0)$ in (9.1) and using the evenness of $g$, we get

$$
\begin{aligned}
\left\|4 g(a x)-4 a^{2} g(x)\right\| & \leq \alpha(x, 0,0), \\
\left\|g(x)-\frac{g(a x)}{n^{2}}\right\| & \leq \frac{1}{4 a^{2}} \alpha(x, 0,0)
\end{aligned}
$$


for all $x \in W$. By using (8.4), for this case $i=0$, it reduces to

$$
\left\|g(x)-\frac{g(a x)}{a^{2}}\right\| \leq \frac{1}{2 a^{2}} \beta(x)
$$

for all $x \in W$. That is,

$$
d(g, T g) \leq \frac{1}{a^{2}} \Rightarrow d(g, T g) \leq \frac{1}{a^{2}}=L=L^{1}<\infty .
$$

Again replacing $x$ by $\frac{x}{a}$ in (8.6), we get

$$
\left\|g(x)-a^{2} g\left(\frac{x}{a}\right)\right\| \leq \frac{1}{4} \alpha\left(\frac{x}{a}, 0,0\right)
$$

for all $x \in W$. That is,

$$
d(g, T g) \leq \frac{1}{2}<1 \Rightarrow d(g, T g) \leq 1=L^{0}<\infty .
$$

In above case, we get $d(g, T g) \leq L^{1-i}$.

The rest of the proof is similar to that of the previous theorem. This completes the proof of the theorem.

Corollary 5. Let $g: W \rightarrow B$ be an even mapping and assume that there exist real numbers $\lambda$ and $s$ such that

$$
\left\|D_{g}\left(x_{1}, x_{2}, x_{3}\right)\right\| \leq \begin{cases}\lambda & \\ \lambda\left(\left\|x_{1}\right\|^{s}+\left\|x_{2}\right\|^{s}+\left\|x_{3}\right\|^{s}\right) ; & s \neq 2 \\ \lambda\left(\left\|x_{1}\right\|^{s}+\left\|x_{2}\right\|^{s}+\left\|x_{3}\right\|^{s}\right)+\left\{\left\|x_{1}\right\|^{3 s}+\left\|x_{2}\right\|^{3 s}+\left\|x_{3}\right\|^{3 s}\right\} ; & s \neq \frac{1}{3}\end{cases}
$$

for all $x_{1}, x_{2}, x_{3} \in X$. Then there exists a unique quadratic mapping $Q: W \rightarrow B$ such that

$$
\left\|g_{q}(x)-Q(x)\right\| \leq\left\{\begin{array}{l}
\frac{\lambda}{4 \mid a^{2}-1} ; \\
\frac{\lambda\|x\|^{s}}{4\left|a^{2}-a^{s}\right|} \\
\frac{\lambda\|x\|^{3 s}}{4\left|a^{2}-a^{3 s}\right|}
\end{array}\right.
$$

for all $x \in X$.

\section{$9 \quad$ Fixed point stability of (1.3): Mixed mapping case}

In this method, we present the Hyers-Ulam stability of the functional equation (1.3) for a mixed mapping case by using fixed point method.

Theorem 10. Let $g: W \rightarrow B$ be a mapping for which there exists a function $\alpha: W^{3} \rightarrow[0, \infty)$ with the condition (7.1) and (8.1) for $a_{i}=\left\{\begin{array}{ll}a & i=0 \\ \frac{1}{a} & i=1,\end{array}\right.$ such that the functional inequality

$$
\left\|D g\left(x_{1}, x_{2}, x_{3}\right)\right\| \leq \alpha\left(x_{1}, x_{2}, x_{3}\right)
$$


for all $x_{1}, x_{2}, x_{3} \in W$. If there exists $L=L(i)$ such that the function

$$
x \rightarrow \beta(x)=\frac{1}{2} \alpha\left(\frac{x}{a}, 0,0\right)
$$

satisfies (7.3) and (8.3) for all $x \in W$, then there exist a unique additive mapping $A: W \rightarrow B$ and a quadratic mapping $Q: W \rightarrow B$ satisfying the functional equation (1.3) and

$$
\|g(x)-A(x)-Q(x)\| \leq \frac{L^{1-i}}{1-L}[\beta(x)+\beta(-x)]
$$

holds for all $x \in W$.

Proof. It follows from (6.2) and Theorem 8 that

$$
\left\|g_{o}(x)-A(x)\right\| \leq \frac{1}{2} \frac{L^{1-i}}{1-L}[\beta(x)+\beta(-x)] .
$$

Similarly, it follows from (7.5) and Theorem 9 that

$$
\left\|g_{e}(x)-Q(x)\right\| \leq \frac{1}{2} \frac{L^{1-i}}{1-L}[\beta(x)+\beta(-x)]
$$

for all $x \in W$. Then $g(x)=g_{o}(x)+g_{e}(x)$ for all $x \in W$.

From (8.11), (9.2) and (9.3), we have

$$
\begin{aligned}
\|g(x)-A(x)-Q(x)\| & =\left\|g_{e}(x)+g_{o}(x)-A(x)-Q(x)\right\| \\
& \leq\left\|g_{o}(x)-A(x)\right\|+\left\|g_{e}(x)-Q(x)\right\| \\
& =\frac{L^{1-i}}{1-L}[\beta(x)+\beta(-x)]
\end{aligned}
$$

for all $x \in W$. Hence the theorem is proved.

Corollary 6. Let $g: W \rightarrow B$ be a mapping and assume that there exist real numbers $\lambda$ and $s$ such that

$$
\left\|D_{g}\left(x_{1}, x_{2}, x_{3}\right)\right\| \leq \begin{cases}\lambda ; & s \neq 1,2 \\ \lambda\left(\left\|x_{1}\right\|^{s}+\left\|x_{2}\right\|^{s}+\left\|x_{3}\right\|^{s}\right) ; & \\ \lambda\left(\left\|x_{1}\right\|^{s}+\left\|x_{2}\right\|^{s}+\left\|x_{3}\right\|^{s}\right)+\left\{\left\|x_{1}\right\|^{3 s}+\left\|x_{2}\right\|^{3 s}+\left\|x_{3}\right\|^{3 s}\right\} ; & s \neq \frac{1}{3}, \frac{2}{3}\end{cases}
$$

for all $x_{1}, x_{2}, x_{3} \in X$. Then there exist a unique additive mapping $A: W \rightarrow B$ and a unique quadratic mapping $Q: W \rightarrow B$ such that

$$
\|g(x)-A(x)-Q(x)\| \leq\left\{\begin{array}{l}
\frac{\lambda}{2|a-1|}+\frac{\lambda}{4\left|a^{2}-1\right|} \\
\frac{\lambda\|x\|^{S}}{2 \mid a-a^{S \mid}}+\frac{\lambda\|x\|^{S}}{4\left|a^{2}-a^{S}\right|} \\
\frac{\lambda\|x\|^{3 S}}{2\left|a-a^{3 S}\right|}+\frac{\lambda\|x\|^{3 S}}{4\left|a^{2}-a^{3 S}\right|}
\end{array}\right.
$$

for all $x \in X$. 


\section{Declarations}

\section{Availablity of data and materials}

Not applicable.

\section{Competing interests}

The authors declare that they have no competing interests.

\section{Fundings}

This work was supported by Basic Science Research Program through the National Research Foundation of Korea funded by the Ministry of Education, Science and Technology (NRF-2017R1D1A1B04032937).

\section{Authors' contributions}

The authors equally conceived of the study, participated in its design and coordination, drafted the manuscript, participated in the sequence alignment, and read and approved the final manuscript. 


\section{References}

[1] J. Aczél and J. Dhombres, Functional equations in several variables, Encyclopedia of Mathematics and its Applications, 31, Cambridge University Press, Cambridge, 1989.

[2] L. Aiemsomboon and W. Sintunavarat, Stability of the generalized logarithmic functional equations arising from fixed point theory, Rev. R. Acad. Cienc. Exactas Fís. Nat. Ser. A Mat. RACSAM 112 (2018), no. 1, 229-238.

[3] T. Aoki, On the stability of the linear transformation in Banach spaces, J. Math. Soc. Japan 2 (1950), 64-66.

[4] I. Chang, E. Lee and H. Kim, On the Hyers-Ulam-Rassias stability of a quadratic functional equations, Math. Inequal. Appl. 6 (2003), 87-95.

[5] K. Ciepliński, Applications of fixed point theorems to the Hyers-Ulam stabiltiy of functional equations - a survey, Ann. Funct. Anal. 3 (2012), 151-164.

[6] St. Czerwik, On the stability of the quadratic mapping in normed spaces, Abh. Math. Sem. Univ. Hamburg 62 (1992), 59-64.

[7] S. Czerwik, Functional equations and inequalities in several variables, World Scientific Publishing Co., Inc., River Edge, NJ, 2002.

[8] A. Ebadian, N. Ghobadipour and M. Eshaghi Gordji, A fixed point method for perturbation of bimultipliers and Jordan bimultipliers in $C^{*}$-ternary algebras, J. Math. Phys. 51 (2010), no. 10, 103508, 10 pp.

[9] V. Govindan, S. Murthy and M. Saravanan, Solution and stability of a cubic type functional equation: Using direct and fixed point methods, Kraguj. J. Math. 44 (2020), 7-26.

[10] P. Găvruţa, A generalization of the Hyers-Ulam-Rassias stability of approximately additive mappings, J. Math. Anal. Appl. 184 (1994), no. 3, 431-436.

[11] O. Hadžić and E. Pap, Fixed point theory in probabilistic metric spaces, Mathematics and its Applications, 536, Kluwer Academic Publishers, Dordrecht, 2001.

[12] D. H. Hyers, G. Isac and T. M. Rassias, Stability of functional equations in several variables, Progress in Nonlinear Differential Equations and their Applications, 34, Birkhäuser Boston, Inc., Boston, MA, 1998.

[13] K. Jun and H. Kim, On the Hyers-Ulam-Rassias stability of a generalized quadratic and additive type functional equation, Bull. Korean Math. Soc. 42 (2005), 133-148. 
[14] S.-M. Jung, Hyers-Ulam-Rassias stability of functional equations in mathematical analysis, Hadronic Press, Inc., Palm Harbor, FL, 2001.

[15] Y. Jung, The Ulam-Gavruta-Rassias stability of module left derivations, J. Math. Anal. Appl. 339 (2008), 108-114.

[16] Pl. Kannappan, Quadratic functional equation and inner product spaces, Results Math. 27 (1995), no. 3-4, 368-372.

[17] Y. Lee, S.-M. Jung and M. T. Rassias. Uniqueness theorems on functional inequalities concerning cubic-quadratic-additive equation. J. Math. Inequal. 12 (2018), no. 1, 43-61.

[18] Y. Lee and G. Kim, Generalized Hyers-Ulam stability of the pexider functional equation, Math. 7 (2019), no. 3, Article No. 280.

[19] M. Mirzavaziri and M. S. Moslehian, A fixed point approach to stability of a quadratic equation, Bull. Braz. Math. Soc. (N.S.) 37 (2006), no. 3, 361-376.

[20] M. S. Moslehian and Th. M. Rassias, Stabiltiy of functional equations in non-Archimedean spaces, Appl. Anal. Discrete Math. 1 (2007), 325-334.

[21] A. Najati and M. B. Moghini, On the stabiltiy of a quadratic and additive functional equation, J. Math. Anal. Appl. 337 (2008), 399-415.

[22] C. Park, Additive $s$-functional inequalities and partial multipliers in Banach algebras, J. Math. Inequal. 13 (2019), no. 3, 867-877.

[23] C. Park, J. R. Lee and X. Zhang, Additive $s$-functional inequality and hom-derivations in Banach algebras, J. Fixed Point Theory Appl. 21 (2019), no. 1, Paper No. 18, 14 pp.

[24] C. Park and T. M. Rassias, Fixed points and stability of the Cauchy functional equation, Aust. J. Math. Anal. Appl. 6 (2009), no. 1, Art. 14, 9 pp.

[25] F. Skof, Proprieta locali e approssimazione di operatori, Rend. Semin. Mat. Fis. Milano 53 (1983), 113-129.

[26] T. M. Rassias, On the stability of the linear mapping in Banach spaces, Proc. Amer. Math. Soc. 72 (1978), no. 2, 297-300.

[27] N. Šrovnik, On certain functional equation in semiprime rings and standard operator algebras, Cubo 16 (2014), no. 1, 73-80.

[28] S. M. Ulam, Problems in modern mathematics, Science Editions John Wiley \& Sons, Inc., New York, 1964. 